\title{
Plantas medicinais utilizadas na comunidade de Campo Preto, Arneiroz, Ceará
}

\section{Medicinal plants used in the community of Campo Preto, Arneiroz, Ceará, Brazil}

\author{
Selma Freire de Brito ${ }^{(D)}{ }^{1}$, Antônio Wanderson Lopes Evangelista ${ }^{(D)}{ }^{2}$ \\ ${ }^{1}$ Doutora em ecologia; professora do Centro de Educação, Ciências e Tecnologia da Região dos Inhamuns, Universidade Estadual do Ceará, Tauá, Ceará; \\ +5585996074647; selma.brito@uece.br. ${ }^{2}$ Graduando em Ciências Biológicas, Centro de Educação, Ciências e Tecnologia da Região dos Inhamuns, Universidade \\ Estadual do Ceará, Tauá, Ceará; ewanderson56@gmail.com.
}

\section{N O T A}

Recebido: $28 / 06 / 2020$

Aprovado: 20/09/2020

Palavras-chave:

Espécies medicinais

Conhecimento popular

Caatinga

Key words:

Medicinal species

Popular knowledge

Caatinga

\begin{abstract}
R E S U M O
A etnobotânica tem o papel de ajudar na compreensão das relações entre o homem e as plantas de uma comunidade. O objetivo desta pesquisa foi realizar um levantamento sobre as plantas medicinais utilizadas na comunidade de Campo Preto, Armeiroz, Ceará, localizada em uma região de Caatinga. Os dados foram obtidos através de uma entrevista previamente preparada que buscava identificar o perfil das pessoas que usam; quais as plantas medicinais utilizadas; as formas de preparo; as partes consumidas; onde essas plantas são obtidas; e para quais doenças são empregadas. Sobre os entrevistados a maioria possui ensino fundamental incompleto e aprendeu sobre plantas medicinais com alguém da família. Foram relatadas 19 plantas, sendo a mais citada Lippia alba. A comunidade recorre ao uso de plantas principalmente para combater resfriados, sendo preparado principalmente na forma de chá. Chama atenção a alta quantidade de espécies exóticas usadas pela população local. Por fim, sabe-se que as plantas medicinais são consumidas por todas as famílias entrevistadas, ressaltando-se a necessidade de conhecer e de incentivar o uso de espécies da flora nativa.
\end{abstract}

A B S T R A C T
Ethnobotany has the role of helping to understand the relationships between men and plants in a
community. The objective of this research was to carry out a survey on medicinal plants used in
the community of Campo Preto, Armeiroz, Ceará, located in a region of Caatinga. The data were
obtained through a previously prepared interview that sought to identify the people who use it;
which medicinal plants are used; the forms of preparation; the parts used; where these plants are
obtained; and what diseases these medicinal plants are used for. Most of the interviewees have
incomplete elementary education and learned about medicinal plants from someone in the family.
Nineteen plants were cited, the most cited being Lippia alba. The community resorts to the use of
plants mainly to fight colds being prepared in the form of tea. It draws attention to the high number
of exotic species used by the community. Finally, it is known that medicinal plants are used by all
interviewed families, emphasizing the need to know and encourage the use of species of native
flora.

Toda essa diversidade vegetal da Caatinga, têm despertado o interesse de pesquisadores que buscam conhecer as espécies com potencial fitoterápico na região (SILVA et al., 2015; REIS; PEREIRA; CANSANÇÃO, 2017; SILVA et al., 2017; ALBERGARIA et al., 2019). Assim, a etnobotânica é uma das maneiras de conhecer as plantas de uma localidade, pois segundo Vishwanathan (2018), consiste no estudo do uso das plantas e na relação do homem com estas. Através destes estudos, conhece-se as formas de uso e manejo das espécies vegetais nas comunidades locais (STRACHULSKI; FLORIANI, 2013). A partir dos levantamentos das potencialidades dos recursos vegetais et al., 2016).

\author{
Revista Verde \\ ISSN 1981-8203 \\ Pombal, Paraíba, Brasil
}

v. 15, n. 4 , out.-dez., p.434-441, 2020

doi: $10.18378 /$ rvads.v15i4.8170 
disponíveis em uma certa comunidade, pode-se planejar também a conservação e a recuperação da área estudada, assim como a otimização dos usos atribuídos as plantas pelos moradores (ROQUE et al., 2010).

Segundo Soldati (2013), o resgate e a manutenção do uso de plantas medicinais são fundamentais para permitir o entendimento da relação entre as pessoas e a natureza. Muitas comunidades rurais conservam forte ligação e dependência dos recursos vegetais locais, pois mesmo com todo o desenvolvimento tecnológico, o cultivo de algumas espécies completam a alimentação e necessidades básicas de sobrevivência das famílias (LUCAS et al., 2015). Da mesma forma, estudos etnobotânicos realizados na Caatinga mostram uma forte relação entre a população dessa região e as plantas medicinais (SILVA et al., 2015; SILVA et al., 2017; LIMANASCIMENTO; RAMOS, 2018; BISPO et al., 2019).

Apesar da importância, muito do conhecimento tradicional sobre plantas medicinais pode desaparecer (ARÉVALO-MARÍN et al., 2015) Baseado em um estudo realizado em uma comunidade rural no semiárido nordestino, Arévalo-Marín et al. (2015) afirmaram que as pressões culturais, a introdução de espécies exóticas e as mudanças geradas pela modernização, podem levar à perda do conhecimento tradicional. Por outro lado, segundo Alencar et al. (2014), as espécies exóticas inseridas dentro de uma comunidade tradicional podem ajudar na resiliência do sistema médico local ou ajudar em papéis que não são executados por espécies nativas.

Portanto, o conhecimento da relação da população com as plantas de uma região torna mais eficiente o planejamento para a conservação, tanto do conhecimento medicinal quanto das espécies vegetais. Assim, o objetivo desta pesquisa foi realizar um levantamento sobre as plantas medicinais utilizadas pela população de Campo Preto, Arneiroz, Ceará, localizado em uma região de Caatinga.

\section{MATERIAL E MÉTODOS}

A presente pesquisa foi desenvolvida na comunidade de Campo Preto, localizada cerca de $28 \mathrm{Km}$ da sede do município de Arneiroz (Latitude: 6 ${ }^{\circ} 19^{\prime}$ 7" Sul; Longitude: 40 9' 44" Oeste). O clima da região é classificado como tropical quente semiárido, com pluviometria média de $506,3 \mathrm{~mm}$ e chuvas concentradas de janeiro a abril. O município possui uma população estimada de 7.302 habitantes, com densidade de 7,07 hab./km² (CEARÁ, 2019).

A comunidade escolhida para o estudo conta com 35 famílias, em sua maioria agricultores familiares e alguns exercem outras atividades como professores e pequenos comerciantes. Essa comunidade foi escolhida através de uma sondagem inicial com a agente comunitária de saúde, que informou que tradicionalmente seus moradores fazem uso e cultivam plantas medicinais. A pesquisa realizada é do tipo qualitativa/quantitativa (PEREIRA; MICLOS, 2013). Isso porque, foi realizado um levantamento acerca de dados quantitativos sobre plantas medicinais, seus nomes populares e uma interpretação do uso dessas espécies.

Para a coleta de dados seguiu-se a metodologia de Gomes et al. (2017), que recomendam que devem ser entrevistados entre $25 \%$ e $50 \%$ da população, em comunidades com até 100 famílias. Nesta pesquisa, 30 famílias (mais de $80 \%$ das casas da comunidade) foram visitadas. Foi considerado como critério de inclusão todas as pessoas acima de 18 anos e que se dispuseram a participar da pesquisa. Além disso, em cada família entrevistouse apenas um membro, que forneceu as informações necessárias para a pesquisa. A escolha do membro para participar da pesquisa foi aleatória de acordo com a presença no momento da visita, disponibilidade em participar da pesquisa e tempo do participante para ceder a entrevista.

A coleta dos dados foi realizada através de uma entrevista semiestruturada. Durante essa entrevista foram feitas perguntas para identificar o perfil das pessoas que utilizam plantas medicinais na comunidade (idade, escolaridade); foram identificados quais são as plantas medicinais mais utilizadas pela comunidade; quais as formas de preparo; quais as partes utilizadas; como foram obtidas, se foram cultivadas em casa ou coletadas em ambiente natural e para quais doenças elas foram utilizadas. Além disso, cada entrevistado recebeu previamente um termo de consentimento de acordo com as normas da Resolução no 466, do Conselho Nacional de Saúde de 12/12/2012 (BRASIL, 2012).

Durante a realização das entrevistas, não foram coletadas amostras das espécies para a herborização. Isto porque, nem todas as espécies apresentavam estruturas reprodutivas no momento da visita ou apresentavam poucas unidades cultivadas apenas para consumo. A identificação das espécies foi realizada então, considerando o nome popular indicado pelos moradores e de fotografias realizadas durante as visitas. Além disso, para a identificação das espécies foram feitas consultas a bibliografias especializadas e as informações disponíveis no banco de dados do Missouri Botanical Garden (TROPICOS, 2019). De posse desses dados, foi pesquisado a família botânica e a forma de crescimento das plantas.

Após a coleta dos dados do perfil da comunidade; da forma de utilização; do preparo e de como o conhecimento foi calculado a frequência relativa (FR) de citação, conforme Magurran (2004), pela seguinte fórmula: $\mathrm{FAi}=(\mathrm{ni} / \mathrm{N}) \times 100(\mathrm{FAi}=$ frequência absoluta $\mathrm{i}$; $\mathrm{ni}=$ número de pessoas que citaram $\mathrm{i} ; \mathrm{N}=$ número total de entrevistados). Todos os cálculos e gráficos foram feitos utilizando o programa SigmaPlot, versão 12.0 (SYSTAT SOFTWARE, 2011).

\section{RESULTADOS E DISCUSSÃO}

Os usuários de plantas medicinais da comunidade Campo Preto em sua maioria, cerca de $75 \%$, são do sexo feminino, com idade variando de no mínimo 30 anos até mais de 80 anos. A pesquisa mostrou que a maior frequência relativa de anos dos usuários foi observada na classe de $39 \mid--49$ anos, com mais de 30\% (Figura 1a). Em uma pesquisa semelhante realizada por Gomes et al. (2017), a faixa etária acima de 50 anos representava cerca de $62,5 \%$ dos entrevistados, mostrando que a faixa etária mais jovem nem sempre está disponível para a realização da entrevista pois, se ausentam mais para atividades como trabalho e escola ou ainda possuem menos informações acerca desta temática. Silva et al. (2015), em um estudo também realizado no Ceará, também entrevistaram em sua maioria pessoas do sexo feminino, com predominância da faixa etária de $36-45$ anos. Isto porque, as mulheres muitas vezes assumem as responsabilidades dos cuidados familiares (OLIVEIRA; LUCENA, 2015). 
Figura 1. Idade (a) e escolaridade (b) dos usuários de plantas medicinais na comunidade de Campo Preto, Arneiroz, Ceará.

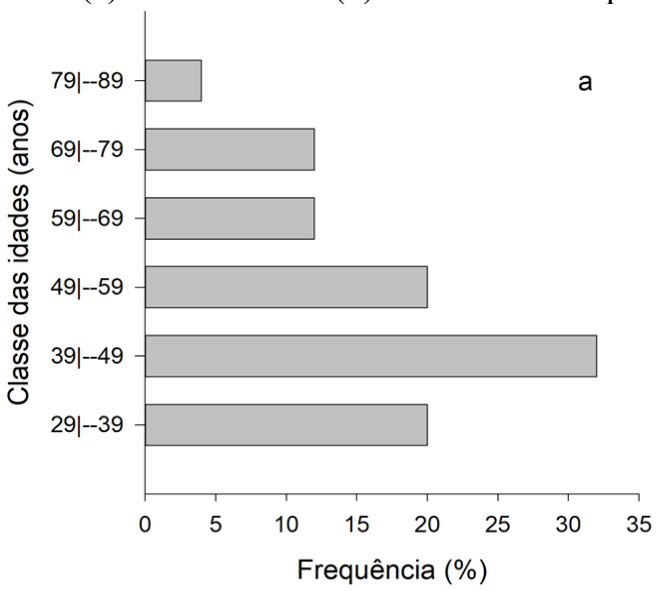

A escolaridade dos usuários de plantas medicinais variou de analfabeto a nível superior completo. Sendo que a maioria, mais de $35 \%$, possuía nível fundamental incompleto. A segunda maior frequência foi observada na classe ensino médio incompleto, com mais de $30 \%$ (Figura 1b). Portanto, na comunidade de Campo Preto, Arneiroz, Ceará, o uso de plantas medicinais é feito por pessoas de diferentes idades e escolaridades. Outros estudos também observam variação na escolaridade e idade, em relação ao uso de plantas medicinais (FIALHO; BASÍLIO; GUSMAN, 2018; BARRETO; SPANHOLI, 2019), mostrando que conhecer

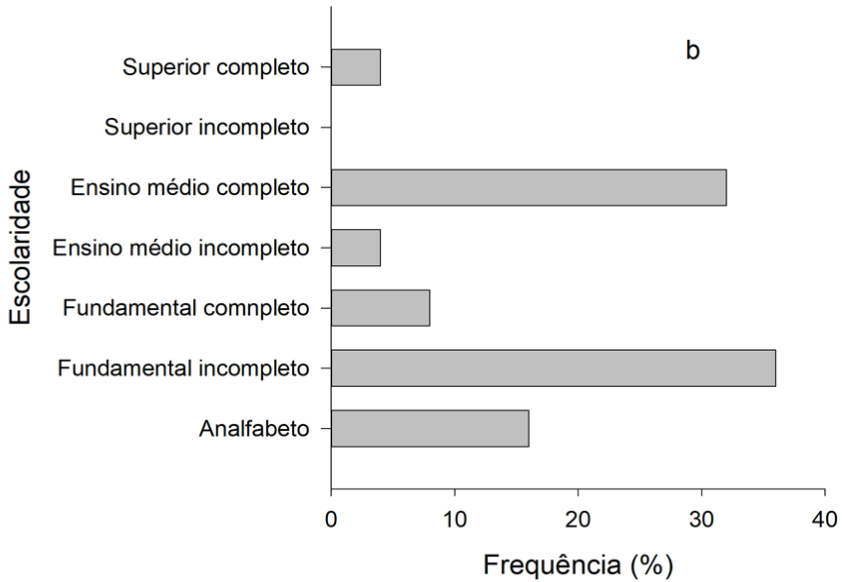

quem usa plantas medicinais é importante para ajudar na conservação dos costumes de uma comunidade.

Todos afirmaram fazer uso de plantas medicinais, sendo que mais de $60 \%$ sempre utilizou. Enquanto, cerca de $15 \%$ afirmaram usar a menos tempo, 5 anos (Figura 2a). Isto mostra que a comunidade interage com os recursos naturais e Bellé (2012), destaca ainda que as plantas medicinais e hortaliças estão relacionadas a qualidade de vida da população, proporcionando bem-estar e mais saúde.

Figura 2. Tempo de uso (a) e origem do conhecimento (b) sobre plantas medicinais na comunidade de Campo Preto, Arneiroz, Ceará.

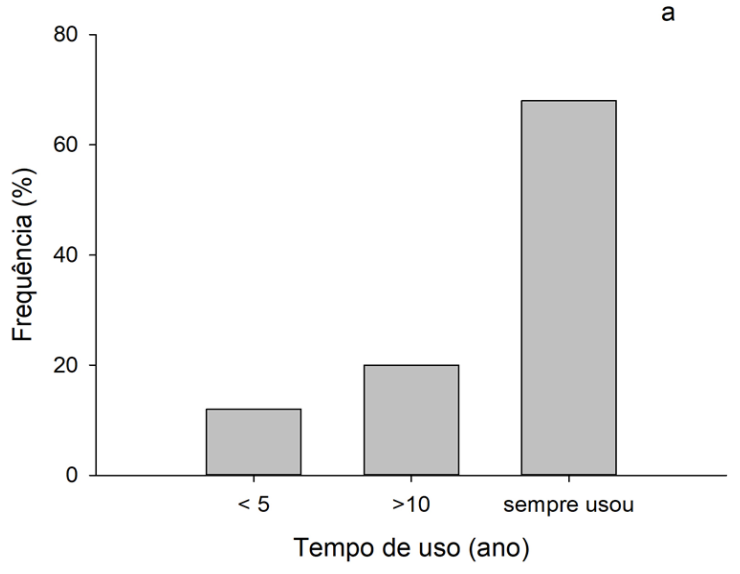

Mais de $90 \%$ da população de Campo Preto, responderam que aprenderam a forma de preparo e indicação de uso com algum familiar e menos de $5 \%$ obtiveram informações com vizinhos (Figura 2b). Nesta linha de pesquisa Cunha et al. (2015), destacaram que o uso popular de plantas medicinais é transmitido oralmente de geração em geração e os estudos etnofarmacológicos auxiliam na preservação destas informações. De acordo com Zuchiwschi et al. (2010), os agricultores tradicionais em suas interações com os recursos naturais, adquirem conhecimentos e estes são transmitidos via experiência pessoal e de forma direta. Assim, como os resultados obtidos nesta pesquisa em sua maioria o conhecimento é passado entre os membros da própria família. Isto ocorre principalmente em comunidades predominantemente agrícola, como é o caso da

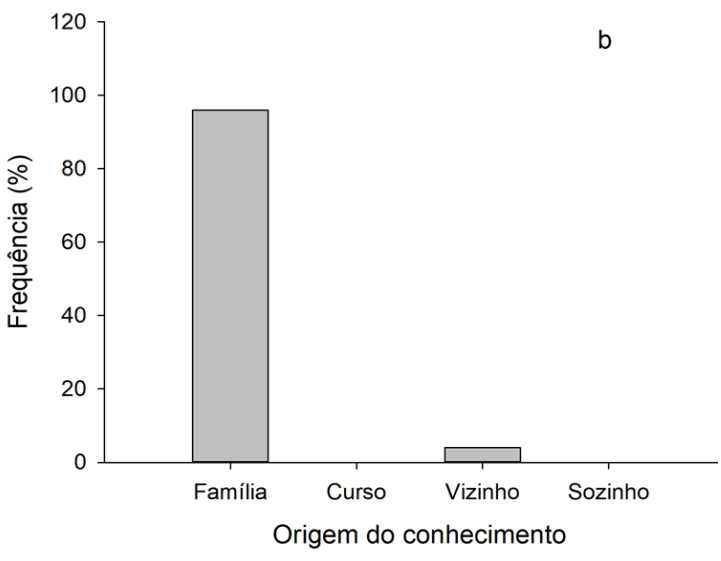

estudada.

Segundo Alves e Povh (2013), a principal fonte de obtenção dos conhecimentos sobre plantas medicinais é por meio de familiares. Isto também foi observado em um estudo realizado por Fialho et al. (2018), onde mais de $70 \%$ dos entrevistados afirmaram ter adquirido o conhecimento com algum familiar. Ressalta-se a importância dos estudos de etnobotânica, pois torna estas informações disponíveis para um maior número de pessoas e contribui para a sua preservação.

Entre as espécies medicinais citadas pelos usuários pode-se destacar Lippia alba (Mill) N. E. Brown, Malva Sylvestre L., Amburana Cearensis Allemão) A.C. Sm e Aloe vera L., sendo mencionado ao todo 19 espécies. Famílias como Lamiaceae e Fabaceae estão entre as que foram citadas (Tabela 1), sendo 
comum encontrar referências a estas famílias em estudos etnobotânicos. Um levantamento das plantas medicinais comercializadas em Juazeiro do Norte, Ceará, Bispo et al. (2019), apontou elevada frequência de citação destas duas famílias. Isto ocorre porque é comum o uso de espécies da família Fabaceae como medicinal na Caatinga (LOIOLA et al., 2010). Além disso, Fabaceae representa grande parte da diversidade florística da Caatinga (BFG, 2015). Enquanto, a família Lamiaceae destacase por apresentar espécies ricas em propriedades medicinais (JUDD et al., 2009).

Tabela 1. Espécies medicinais utilizadas na comunidade de Campo Preto, Arneiroz, Ceará.

\begin{tabular}{|c|c|c|c|c|c|}
\hline Nome científico & $\begin{array}{l}\text { Nome } \\
\text { popular }\end{array}$ & Família botânica & Ação & Hábito & Origem \\
\hline Lippia alba (Mill) N. E. Brown & Erva cidreira & Verbenaceae & Calmante, dor & Herbáceo & Nativa \\
\hline Malva sylvestre $\mathrm{L}$. & Malva & Malvaceae & $\begin{array}{l}\text { Anti-inflamatório, } \\
\text { resfriados }\end{array}$ & Herbáceo & Exótica \\
\hline $\begin{array}{l}\text { Amburana cearensis (Allemão) A.C. } \\
\text { Sm }\end{array}$ & Amburana & Fabaceae & Anticoagulante & Árvore & Nativa \\
\hline Citrus limon (L.) Burmann F. & Limão & Rutaceae & $\begin{array}{l}\text { Doenças infecciosas, } \\
\text { resfriados }\end{array}$ & Arbusto & Exótica \\
\hline Aloe vera $\mathrm{L}$. & Babosa & Asphodelaceae & Cortes, queimaduras & Herbáceo & Exótica \\
\hline $\begin{array}{l}\text { Caesalpinia ferrea Mart. ex. Tul. } \\
\text { var. ferrea }\end{array}$ & Pau-ferro & Fabaceae & Bronquite, inflamações & Árvore & Nativa \\
\hline Myracrodruon urundeuva Allemão & Aroeira & Anacardiaceae & Doenças sexuais & Árvore & Nativa \\
\hline Cymbopogon citratus (DC.) Stapf. & Capim santo & Poaceae & Cólicas, calmante & Herbáceo & Exótica \\
\hline Mentha $\mathrm{x}$ villosa Huds. & Hortelã & Lamiaceae & Dores de cabeça & Herbáceo & Exótica \\
\hline Waltheria indica $\mathrm{L}$. & $\begin{array}{l}\text { Malva-do- } \\
\text { reino }\end{array}$ & Malvaceae & Anti-inflamatório & Herbáceo & Exótica \\
\hline Achyrocline satureioides (Lam) D.C & Macela & Asteraceae & $\begin{array}{l}\text { Dores de estômago; } \\
\text { cólica }\end{array}$ & Herbáceo & Nativa \\
\hline Citrus sinensis (L.) Osbeck & Laranja & Rutaceae & Calmante & Árvore & Exótica \\
\hline Punica granatum $\mathrm{L}$. & Romãn & Punicaceae & Diarreia, garganta & Arbusto & Exótica \\
\hline Ximenia americana $\mathrm{L}$. & $\begin{array}{l}\text { Ameixa } \\
\text { silvestre }\end{array}$ & Olacaceae & Diabetes & Arvore & Exótica \\
\hline $\begin{array}{l}\text { Sansevieria } \quad \text { trifasciata } \\
\text { Laurenttii }\end{array}$ & $\begin{array}{l}\text { Espada de } \\
\text { são Jorge }\end{array}$ & Liliaceae & Dores de estômago & Herbáceo & Exótica \\
\hline $\begin{array}{l}\text { Brassica integrifólia (H. West) } \\
\text { Rupr. }\end{array}$ & Mostarda & Brassicaceae & Tratamento do ma-hálito & Arbusto & Exótica \\
\hline Anacardium occidentale $\mathrm{L}$. & Cajueiro & Anacardiaceae & $\begin{array}{l}\text { Irritação na pele; } \\
\text { infecções }\end{array}$ & Árvore & Exótica \\
\hline Eucalyptos globulus Labill & Eucalipto & My & Febre, resfriados & Árvore & Exótica \\
\hline Chenopodium ambrosioides $\mathrm{L}$. & Mastruz & Amaranthaceae & $\begin{array}{l}\text { Anti-inflamatório, } \\
\text { pancadas }\end{array}$ & Herbáceo & Nativa \\
\hline
\end{tabular}

Das 19 espécies, nove apresentam porte herbáceo, sete são árvores e três arbustos (Tabela 1), havendo predominância das espécies de porte herbáceo, o que pode estar associado ao requerimento de pequenos espaços para o cultivo. Este resultado também foi observado por Aguiar e Barros (2012), no interior do Piauí e por Rodrigues e Andrade (2014), no interior de Pernambuco, onde a maioria das espécies citadas nos levantamentos apresentavam porte herbáceo.

Merece destaque a grande quantidade de espécies exóticas utilizadas para fins medicinais. De acordo com os dados, mais de $68 \%$ das espécies são exóticas. Em um estudo de Gomes et al. (2017), das 25 espécies catalogadas durante entrevista a moradores, 16 delas eram exóticas e apenas 9 nativas. Isso mostra que assim como neste estudo, muitas das espécies medicinais identificadas através de levantamentos etnobotânicos são exóticas.

Lima-Nascimento et al. (2018), também destacam a intensa ocorrência de espécies exóticas usada pela população em uma comunidade inserida na região de Caatinga de Pernambuco e como isto pode acarretar danos ao ecossistema. Espécies exóticas devem ser evitadas, pois podem se tornar invasoras de áreas naturais (KÜSTER et al., 2012) e o uso das espécies nativas deve ser mais valorizado, pois são mais conhecidas pela população local. Contudo, Alencar et al. (2014), ressaltam que o uso de espécies exóticas pode ajudar na conservação das espécies nativas e a suprir finalidades medicinais que não são atendidas por espécies nativas. Portanto, cada caso deve ser avaliado individualmente e assim planejado ações de conservação. Para a comunidade de Campo Preto, sugere-se ações de divulgação e incentivo do uso de espécies nativas.

Das 19 espécies, a erva-cidreira foi citada com mais frequência, mais de $30 \%$. Outras espécies como a babosa, o capim santo, hortelã, malva do reino e laranja também foram frequentemente citadas pelos usuários, com mais de $5 \%$ das citações (Figura 3). A erva-cidreira é uma espécie que apresenta comprovada eficácia medicinal (GAZOLA et al., 2004). Além 
disso, já foi mencionada em outros levantamentos etnobotânicos na Caatinga (REIS et al., 2017; BISPO et al., 2019). Um estudo de Brito; Senna-Valle (2011), mostrou que a erva-cidreira foi a espécie indicada para o tratamento de um maior número de sintomas. A variação da frequência de uso das plantas medicinais em comunidades rurais, é relacionado, as diferentes necessidades de tratamento, além disso uma mesma planta pode estar sendo utilizada para mais de uma doença e assim ser citada mais vezes (RIBEIRO et al., 2014).

Figura 3. Frequência de uso das espécies medicinais na comunidade de Campo Preto, Arneiroz, Ceará.

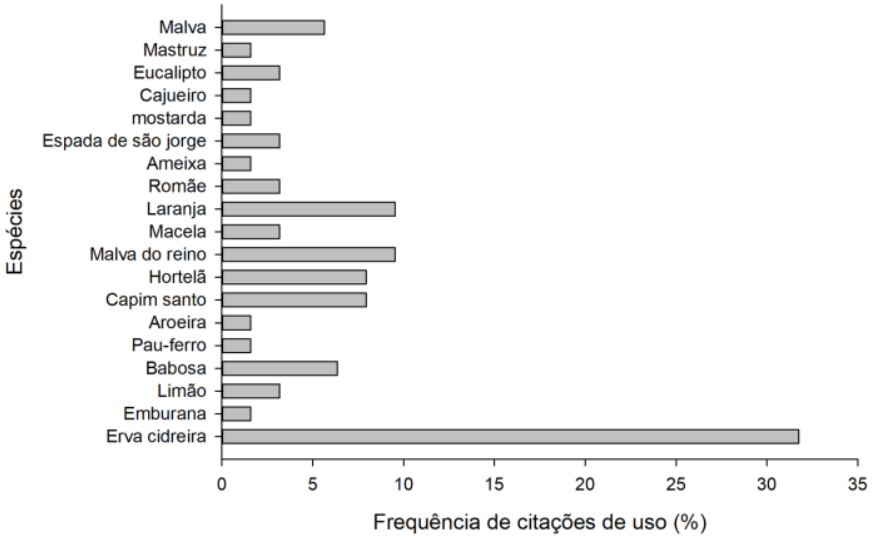

Cerca de $33 \%$ dos usuários afirmaram fazer uso de plantas medicinais para combater resfriados. Enquanto, doenças como dor de cabeça, calmante e febre aparecem na sequência como as mais citadas (Figura 4). Uma pesquisa realizada acerca do uso de plantas medicinais na comunidade de São João Da Várgea em Mossoró - RN, mostrou que a maioria dos entrevistados fazem uso de plantas medicinais para tratar doenças do sistema respiratório e digestório, principalmente as que combatem os sintomas de gripe (FREITAS et al., 2015). Isso pode indicar que as plantas medicinais são utilizadas para enfermidades mais simples. Contudo, também mostra que é necessário que se planeje ações voltadas para a educação em saúde, uma vez que além de orientar o uso das plantas, pode somar experiências, repassar o conhecimento tradicional e fornecer bases para novas pesquisas (MADEIRO; LIMA, 2015).

Figura 4. Doenças tratadas com plantas medicinais pela população da comunidade de Campo Preto, Arneiroz, Ceará.

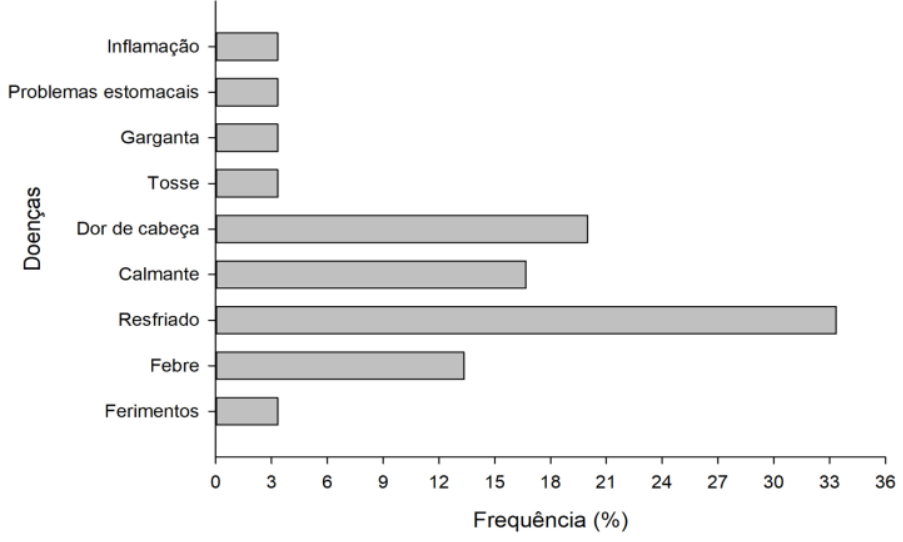

Sobre as partes mais utilizadas das plantas medicinais, a pesquisa indica que mais de $80 \%$ da população faz o uso somente das folhas. Enquanto, outras partes como frutos, casca e sementes também foram citados, com frequência de menos de $10 \%$ (Figura 5a). Resultados obtidos por Oliveira et al. (2010), mostram que utilização das folhas correspondeu a $31,5 \%$ das citações da pesquisa. Isto pode indicar ainda uma maior disponibilidade das folhas, já que flores, frutos e sementes não se encontram disponíveis em todas as épocas do ano (VÁSQUEZ et al., 2014). Além disso, nas folhas são encontrados a maior parte dos princípios ativos das plantas (SANTOS-LIMA et al., 2016). Silva et al. (2012) e Lisboa et al. (2017), em suas pesquisas etnobotânicas relataram também que as folhas são a parte da planta mais utilizadas nos preparos com plantas medicinais.

Figura 5. Parte utilizada (a) e forma de preparo (b) das plantas medicinais usadas pela população da comunidade de Campo Preto, Arneiroz, Ceará.
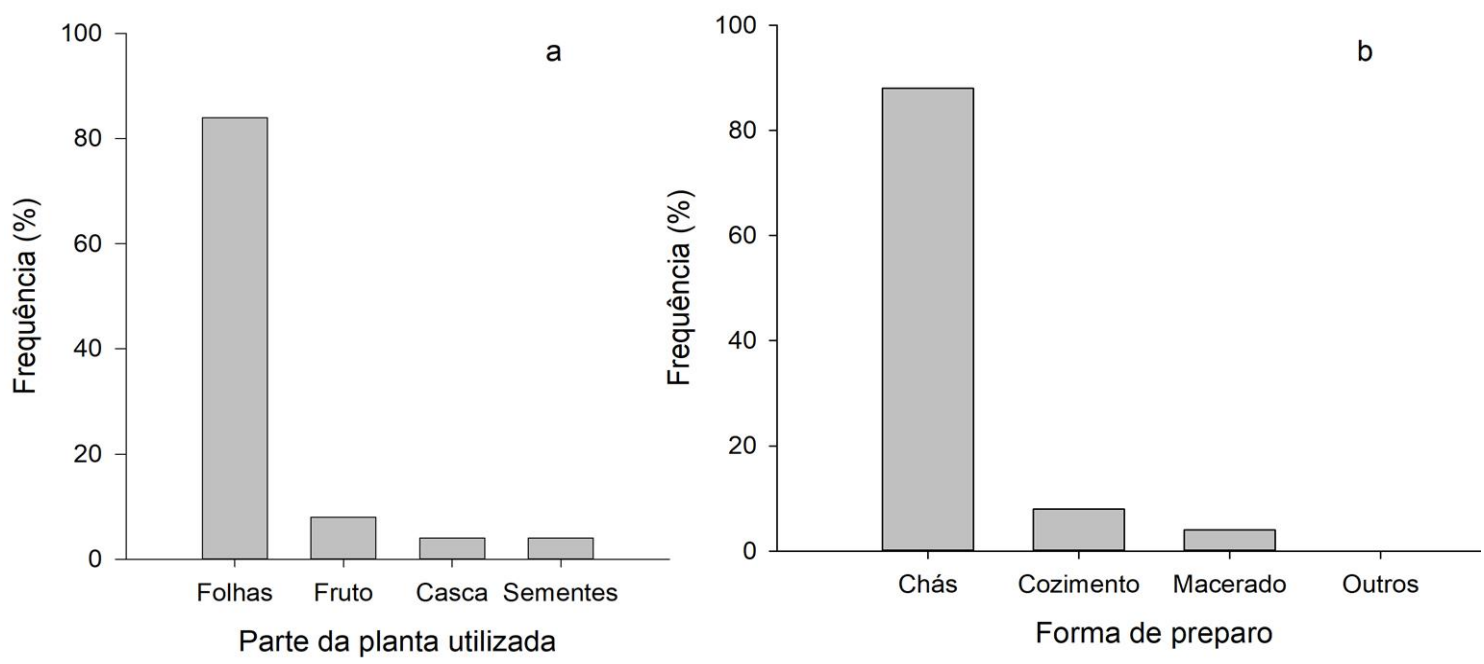

Revista Verde, v.15, n.4, p.434-441, 2020 
A maioria da população, mais de $80 \%$, fazem uso das plantas medicinais forma de chá. Cozimento e macerados também foram citados pelos entrevistados, com cerca de $10 \%$ (Figura 5b). As formas de preparo das plantas medicinais dependem, tanto da espécie em questão, quanto da enfermidade a ser tratada (ARAÚJO et al., 2014). Segundo Amorozo (2012), as práticas relacionadas ao uso popular de plantas medicinais são o que muitas comunidades têm como alternativa viável para o tratamento de doenças ou manutenção da saúde. Silva et al. (2015), em um levantamento etnobotânico também realizado no Ceará destacou o chá como forma de preparo citada pelos entrevistados.

\section{CONCLUSÕES}

Na comunidade de Campo Preto, Arneiroz, Ceará, todos os moradores fazem uso de plantas medicinais, sendo a espécie mais usada a Lippia alba. Resfriado é a doença mais tratada com plantas. A principal forma de preparo é o chá, sendo a parte mais utilizada as folhas.

A maioria das plantas medicinais usada pela comunidade são exóticas. Alertando para a necessidade de valorização e resgate das espécies nativas da flora.

\section{REFERÊNCIAS}

AGUIAR, L. C. G. G.; BARROS, R. F. M. Plantas medicinais cultivadas em quintais de comunidades rurais no domínio do cerrado piauiense (Município de Demerval Lobão, Piauí, Brasil). Revista Brasileira de Plantas Medicinais, v.14, n.3, p.419-434, 2012. 10.1590/S1516-05722012000300001.

AlBERGARIA, E. T.; SILVA, M. V.; SILVA, A. G. Levantamento etnobotânico de plantas medicinais em comunidades rurais localizadas na Unidade de Conservação Tatu-Bola, município de Lagoa Grande, PE - Brasil. Revista Fitos, v.13, n.2, p.137-154, 2019. 10.17648/2446-4775.2019.713.

ALENCAR, N. L.; SANTOROA, F. R.; ALBUQUERQUE, U. P. What is the role of exotic medicinal plants in local medical systems? A study from the perspective of utilitarian redundancy. Revista Brasileira Farmacogn, v.24, p.506-515, 2014. $\underline{10.1016 / \text { j.bjp.2014.09.003. }}$.

ALVES, G. S. P.; POVH, J. A. Estudo etnobotânico de plantas medicinais na comunidade de Santa Rita, Ituiutaba - MG. Biotemas, v. 26, p. 231-242, 2013. 10.5007/21757925.2013v26n3p231.

AMOROZO, M. C. M. Uso e diversidade de plantas medicinais em Santo Antônio do Leverger, MT, Brasil. Atlas Botânica Brasílica, v. 16, n. 2, p.189-203, 2012. 10.1590/S010233062002000200006.

ARAÚJO, C. R. F. de; SILVA, A. B.; TAVARES, E. C.; COSTA, E. P. da.; MARIZ, S. R. Perfil e prevalência de uso de plantas medicinais em uma unidade básica de saúde da família em Campina Grande, Paraíba, Brasil. Revista de Ciências Farmacêuticas Básica e Aplicada, v. 35, n. 2, p.233-38, 2014.
ARÉVALO-MARÍN, E.; LIMA, J. R. F.; PALMA, A. R. T.; LUCENA, R. F. P.; CRUZ, D. D. Traditional Knowledge in a Rural Community in the Semi-Arid Region of Brazil: Age and gender patterns and their implications for plant conservation. Ethnobotany Researchand Applications, v.14, p.331-344, 2015. 10.17348/era.14.0.331-344

BARRETO, M. R.; SPANHOLI, M. L. Estudo etnobotânico em comunidades rurais de Sinop, Mato Grosso, Brasil. Interações (Campo Grande), v.20, n.1, p. 267-282, 2019. 10.20435/inter.v20i1.1889.

BFG. 2015. Growing knowledge: an overview of Seed Plant diversity in Brazil. Rodriguésia 66: 1085-1113

BELLÉ, S. Plantas Medicinais: caracterização, cultivo e uso paisagístico na Serra Gaúcha. Bento Gonçalves; editora IFRSCampus bento Gonçalves, 2012. 200p.

BRASIL. Ministério da Saúde. Conselho Nacional de Saúde. Resolução n. 466, de 12 de dezembro de 2012. Aprova diretrizes e normas regulamentadoras de pesquisas envolvendo seres humanos. Brasília, Diário Oficial da União, 2012.

BISPO, G. L.; MARCO, C. A.; FERREIRA, F. S.; CHAVES, J. T. L.; FARIAS, R. B. Estudo etnobotânico de plantas medicinais no comércio da cidade de Juazeiro do Norte, CE. Journal of Biology \& Pharmacy and Agricultural Management, v.15, n.4, p.450-465, 2019.

BRITO, M. R.; SENNA-VALLE, L. de. Plantas medicinais utilizadas na comunidade caiçara da Praia do Sono, Paraty, Rio de Janeiro, Brasil. Acta Botanica Brasilica, v.25, n.2, p.363-372, 2011. 10.1590/S0102-33062011000200012.

CEARÁ, Instituto de Pesquisa e Estratégia Econômica do Ceará IPECE. Disponível em: $<$ https://www.ceara.gov.br/2010/03/17/municipios-com-a-letraa/\#munic-pio-arneiroz.> Acessado em: 15 de dezembro 2019.

CUNHA, M. M. C.; GONDIM, R. S. D.; BONFIM, B. F.; BATALHA JUNIOR, N. J. P.; BARROSO, W. A.; VILANOVA, C. M. Perfil etnobotânico de plantas medicinais comercializadas em feiras livres de São Luís, Maranhão, Brasil. Scientia Plena, v. 11, 12, p. 2-12, 2015. 10.14808/sci.plena.2015.121202.

FERNANDES, M. F; QUEIROZ, L. P. Vegetação e flora da Caatinga. Ciência e Cultura, v.70, n.4, p.51-56, 2018. 10.21800/2317-66602018000400014.

FIALHO, J. R.; BASÍliO, L. C.; GUSMAN, G. S. Perfil Socioeconômico e Etnobotânico em Posto do Programa da Saúde da Família (Psf) em Viçosa-Minas Gerais. Revista Científica Univiçosa, v.10, n.1, p.899-905, 2018.

FREITAS, A.V. L.; COELHO, M. F. B.; PEREIRA, Y. B.; FREITAS NETO, E. C.; AZEVEDO, R. A. B. Diversidade e usos de plantas medicinais nos quintais da comunidade de São João 
Da Várzea em Mossoró, Rio Grande Do Norte. Revista Brasileira de Plantas medicinais, v.17, n.4, p. 845-856, 2015. $\underline{10.1590 / 1983-084 X / 14080 .}$.

GAZOLA, R.; MACHADO, D.; RUGGIERO, C.; SINGI, G.; ALEXANDRE, M. M. Lippia alba, Melissa officinalis and Cymbopogon citratus: effects of the aqueous extratcts on the isolated hearts of rats. Pharmacol research, v.50, p. 477-480, 2004. 10.1016/j.phrs.2004.01.012.

GOMES, T. M. F.; LOPES, J. B.; BARROS, R. F. M.; ALENCAR, N. L. Plantas de uso terapêutico na comunidade rural Bezerro morto, são João da cana Brava, Piauí, Brasil. Gaia Scientia, v.11, n.1, p.253-268, 2017. 10.22478/ufpb.1981$\underline{1268.2017 \mathrm{v} 11 \mathrm{n} 1.33683}$.

JUDD, W. S.; CAMPBELL, C. S.; KELLOGG, E. A.; STEVENS, P. F.; DONOGHUE, M. J. Sistemática Vegetal: Um Enfoque Filogenético. 3. ed. Porto Alegre: Artmed, 2009. 632 p.

KÜSTER, L. C.; STEDILLE, L. I. B.; DACOREGIO, H.; SILVA, A. C. da; HIGUCHI, P. Avaliação de riscos e procedência de espécies arbóreas nas escolas estaduais de Lages, SC. Revista de Ciências Agroveterinárias, v.11, n.2, p.118-125, 2012.

LIMA-NASCIMENTO, A. M.; BENTO-SILVA, J. S.; RAMOS, E. M. N. F. Conhecimento e uso das plantas da caatinga por agricultores locais moradores de uma comunidade rural do Município de Pesqueira Estado de Pernambuco. CIENTECRevista de Ciência, Tecnologia e Humanidades do IFPE, v.10, n.1, p.75-91, 2018.

LISBOA, M. S. Estudo etnobotânico em comunidade quilombola Salamina, Putumujú em Maragogipe, Bahia. Revista Fitos, v.11, n.1, p.1-118, 2017. 10.5935/2446-4775.20170006.

LOIOLOA, M. I. B.; PATERNO, G. B. C.; DINIZ, J. A.; CALADO, J. F.; OLIVEIRA, A. C. P. Leguminosas e seu potencial de uso em comunidades rurais de São Miguel do Gostoso-RN. Revista Caatinga, v. 23, p. 59-70, 2010.

LUCAS F. C. A; LEÃO, V. M.; REIS, A. S. dos; TAVARES MARTINS, A. C. C.; COSTA, J. C. M. da. Usos e benefícios das plantas em comunidades rurais de Capanema, Pará, Brasil. Cadernos de Agroecologia, v.10, n.3, p.1-5, 2015.

MADEIRO, A. A. S.; LIMA, C. R. L. Estudos etnofarmacológicos de plantas medicinais utilizadas no brasil: revisão de literatura. Ciências Biológicas e da Saúde, v. 3, n.1, p. 69-76, 2015.

MAGURRAN, A. E. 2004. Measuring biological diversity. Oxford, Blackwell Science, 256p.

OLIVEIRA, F. C. S.; BARROS, R. F. M.; MOITA NETO, J. M. Plantas medicinais utilizadas em comunidades rurais de Oeiras, semiárido piauiense. Revista Brasileira de Plantas medicinais, v.12, n.3, p.282-301, 2010. 10.1590/S1516-05722010000300006
OLIVEIRA, D. M. S.; LUCENA, E. M. P. O uso de plantas medicinais por moradores de Quixadá-Ceará. Revista Brasileira de Plantas Medicinais, v. 17, n. 3, p. 107-412, 2015. $\underline{10.1590 / 1983-084 X / 13 \quad 095}$.

PEREIRA, K. R.; MICLOS, P. V. Pesquisa Quantitativa e Qualitativa: A integração do conhecimento científico. Saúde \& Transformação Social, v.48, p.16-18, 2013.

REIS, C. R. M.; PEREIRA, A. F. N.; CANSANÇÃO, I. F. Levantamento Etnobotânico de Plantas Medicinais Utilizadas por Moradores do Entorno do Parque Nacional Serra da Capivara - PI. Journal of Biology \& Pharmacy and Agricultural Management, v.13, n.4, 2017.

RIBEIRO, D. A.; MACÊDO, D. G; OLIVEIRA, L. G. S.; SARAIVA, M. E.; OLIVEIRA, S. F.; SOUZA, M. M. A.; MENEZES, I. R. A. Therapeutic potential and use of medicinal plants in an area of the Caatinga in the state of Ceará, northeastern Brazil. Revista Brasileira de Plantas Medicinais, v.16, n.4, p.1-10, 2014. 10.1590/1983-084X/13 059.

RODRIGUES, A. P.; ANDRADE, L. H. C. Levantamento etnobotânico das plantas medicinais utilizadas pela comunidade de Inhamã, Pernambuco, Nordeste do Brasil. Revista brasileira de plantas medicinais, v.16, n.3, p.721-730, 2014. 10.1590/1983$\underline{084 x / 08 \quad 159}$.

ROQUE, A. A.; ROCHA, R. M.; LOIOLA, M. I. B. Uso e diversidade de plantas medicinais da Caatinga na comunidade rural de Laginhas, município de Caicó, Rio Grande do Norte (Nordeste do Brasil). Revista Brasileira de Plantas Medicinais, v.12, n.1, p.31-42, 2010. 10.1590/S1516-05722010000100006.

SANTOS-LIMA, T. M.; SANTOS, D. R. V.; SOUZA, R. M.; BASTOS, N. G.; VANNIER-SANTOS, M. A.; NUNES, E. S.; DIAS-LIMA, A. G. Plantas medicinais com ação antiparasitária: conhecimento tradicional na etnia Kantaruré, aldeia Baixa das Pedras, Bahia, Brasil. Revista brasileira de plantas medicinais, v.18, n.1, p.240-247, 2016. 10.1590/1983-084X/15_063.

SILVA, N. C. B.; REGIS, A. C. D.; ALMEIDA, M. Z. Estudo Etnobotânico em Comunidades Remanescentes de Quilombo em Rio de Contas, Chapada Diamantina, Bahia. Revista Fitos, v.7, n.2, p.1-11, 2012.

SILVA, S.; ANSELMO, M. G. V.; DANTAS, W. M.; ROSA, J. H.; NUNES, E. N.; SOARES, J. P.; ALVES, C. A. B. Conhecimento e Uso de Plantas Medicinais em uma Comunidade Rural no Município de Cuitegi, Paraíba, Nordeste do Brasil. Revista Gaia Scientia, v.8, n.1, p.248-265, 2014.

SILVA, C. G.; MARINHO, M. G. V.; LUCENA, M. F. A.; COSTA, J. G. M. Levantamento etnobotânico de plantas medicinais em área de Caatinga na comunidade do Sítio Nazaré, município de Milagres, Ceará, Brasil. Revista Brasileira de Plantas Medicinais, v.17, n.1, p.133-142, 2015. 10.1590/1983$\underline{084 \mathrm{X} / 12055 .}$ 
SILVA, M. O. M.; MOREIRA, L. M. C. C.; FELISMINO, D. C. Levantamento Etnofarmacológico de Espécies Medicinais em Área da Reserva Florestal de Caatinga no Município de Santa Cruz do Capibaribe, PE. Journal of Biology \& Pharmacy and Agricultural Management, v.13, n.1, p.35-40, 2017.

SOLDATI, G. T. 2013. Transmissão de conhecimento: origem social das informações e evolução cultural. In: ALBUQUERQUE, U.P. (org.) Etnobiologia: bases ecológicas e evolutivas. Recife, PE: NUPEEA. p. 37-61.

SOUSA, F. Q. S.; ANDRADE, L. A.; XAVIER, K. R. F. Cryptostegia madagascariensis Bojer ex Decne.: impactos sobre a regeneração natural em fragmentos de caatinga. Revista Brasileira de Ciências Agrárias, v.11, n.1, p. 39-45, 2016. $\underline{10.5039 / a g r a r i a . v 11 i 1 a 5357 .}$

STRACHULSKI J.; FLORIANI, N. Conhecimento popular sobre plantas: um estudo etinobotânico na comunidade rural na linha Criciumal, em Cândido de Abreu-PR. Revista Geografar, v.8, n.1, p.125-153, 2013.

SYSTAT SOFTWARE. SigmaPlot for Windows Version 12.0. San Jose: Systat Software Inc., 2011.

TROPICOS.org. Missouri Botanical Garden. Disponível em: <http://www.tropicos.org. $>$ Acesso em 13 dezembro 2019.

VÁSQUEZ, S. P. F.; MENDONÇA, M. S.; NODA, S. N. Etnobotânica de plantas medicinais em comunidades ribeirinhas do Município de Manacapuru, Amazonas, Brasil. ACTA AMAZONICA, v.44, n.4, p. 457-472, 2014. 10.1590/1809$\underline{4392201400423}$.

VISHWANATHAN, A. S. Ethnobotany: A Bridge Between Traditional Knowledge and Biotechnological Studies on Medicinal and Aromatic Plants: Conservation, Genetic Improvement and Utilization. In: Kumar, N. Biotechnological Approaches for Medicinal and Aromatic Plants, pp.383-394. 2018.

ZUCHIWSCHI, E.; FANTINI, A. C.; ALVES, A. C.; PERONI, N. Limitações ao uso de espécies florestais nativas pode contribuir com a erosão do conhecimento ecológico tradicional e local de agricultores familiares. Acta Botanica Brasilica, v. 24, n. 1, p. 270-282, 2010. 10.1590/S0102-33062010000100029. 